\title{
STOPPED-FLOW SPECTROPHOTOMETRIC STUDIES OF THE KINETICS OF INTERACTION OF DIHYDROXYFUMARIC ACID WITH THE DPPH FREE RADICAL
}

\author{
Natalia Secara \\ Institute of Chemistry of the Academy of Sciences of Moldova, 3 Academiei str., MD 2028, Chisinau, Moldova \\ E-mail: natalia_secara@yahoo.com,Phone: 729761
}

\begin{abstract}
The reaction of dihydroxyfumaric acid with the free radical 2,2-diphenyl-1-picrylhydrazyl (DPPH) was studied using the stopped-flow method, in order to describe the reaction kinetics. Dihydroxyfumaric acid reacts very rapidly with DPPH, the reaction being completed in several minutes. This 2-stoichiometric reaction proceeds in two stages, with reaction orders of 1 and 0.76 with respect to DPPH, and 0.5 and 0.3 with respect to DHF, respectively. The rate constant of the two stages of the reaction were found to be $39.1(\mathrm{~L} / \mathrm{mol} \cdot \mathrm{s})$ and $0.0012\left(\mathrm{~s}^{-1}\right)$ at $20^{\circ} \mathrm{C}$ and $\mathrm{pH} 4.0$.
\end{abstract}

Keywords: DPPH; antioxidant activity; stoichiometry; stopped-flow; kinetics; dihydroxyfumaric acid

\section{Introduction}

Many studies have reported the carcinogenic and mutagenic implications of oxidative stress or excessive production of reactive oxygen species, resulting in DNA damage [1], lipid peroxidation [2], depletion of protein sulfhydryl groups, glutathione levels [3] and other negative effects. Free radical-mediated tissue damage is generally accepted as a major mechanism underlying the occurrence of certain chronic diseases [4].

Antioxidants play an important role in the protection of the cell from oxidants attack by donating hydrogen atoms to the active radicals, while the radicals formed from the antioxidant molecules are stable species that stop the oxidation chain reaction [5]. One of such antioxidants is the dihydroxyfumaric acid (DHF), produced by slow oxidation of tartaric acid. The DHF plays a very important role in nature, as it represents an intermediate compound in the biosynthesis of 3-phosphoglicerinic acid. Also, due to its reducing properties, DHF enhances the taste and odor of wine [6].

In order to estimate the antioxidant activity of many compounds, the DPPH" method has been widely applied during recent years. DPPH' is a stable nitrogen-centered free radical by virtue of the delocalization of the spare electron over the molecule as a whole, so that the molecules do not dimerise, as would be the case of most other molecules [7].

A quantitative analysis of the $\mathrm{H}$-atom transfer reaction from a given antioxidant to $\mathrm{DPPH}^{*}$ provides a quite simple way to characterize the reaction kinetics. The $\mathrm{H}$-transfer reactions are monitored by recording the decay of the DPPH* visible absorption band $\left(\lambda_{\max }=520 \mathrm{~nm}\right.$ in $70 \%$ methanol at $\left.\mathrm{pH} 4.0\right)$, which reflects the transformation of the DPPH radical into the corresponding hydrazine (DPPH-H) by the antioxidant compound.

In this paper, we explore the kinetic behavior of DHF, in order to build a plausible kinetic model and better understand the mechanism of its interaction with DPPH:

\section{Materials and methods}

Reagents

Methanol (>99,8\%) was purchased from Fluka. 2,2-Diphenyl-1-picrylhydrazyl (DPPH') was obtained from Sigma and dihydroxyfumaric acid (98\%) was from Aldrich.

\section{Spectrophotometric measurements}

The H-atom transfer reactions were monitored using an E-100 stopped-flow spectrophotometer (KinTek Corporation, USA). The exact absorbance of each solution was registered before each run using a Lambda 25 UV/VIS spectrophotometer (Perkin Elmer), using $10 \mathrm{~mm}$ quartz cuvettes. All spectrophotometric data were obtained at room temperature $\left(20^{\circ} \mathrm{C}\right)$.

\section{Kinetics of reduction of $D P P H^{\circ}$ by $D H F$}

In order to determine the reaction stoichiometry, different molar ratios, expressed as moles of antioxidant per mole of $\mathrm{DPPH}^{*}$, were tested, ranging from 0.1 to 10 . For each molar ratio, the remaining concentration of $\mathrm{DPPH}^{*}$ at the plateau was determined and graphed, and $\mathrm{EC}_{50}$ was read on the graph as the molar ratio which reduces half of the initial $\mathrm{DPPH}^{*}$ concentration, was determined from the graph. The number of reduced $\mathrm{DPPH}^{*}$ molecules per one molecule of antioxidant was defined as $\sigma=1 /\left(2 \times \mathrm{EC}_{50}\right)$. 


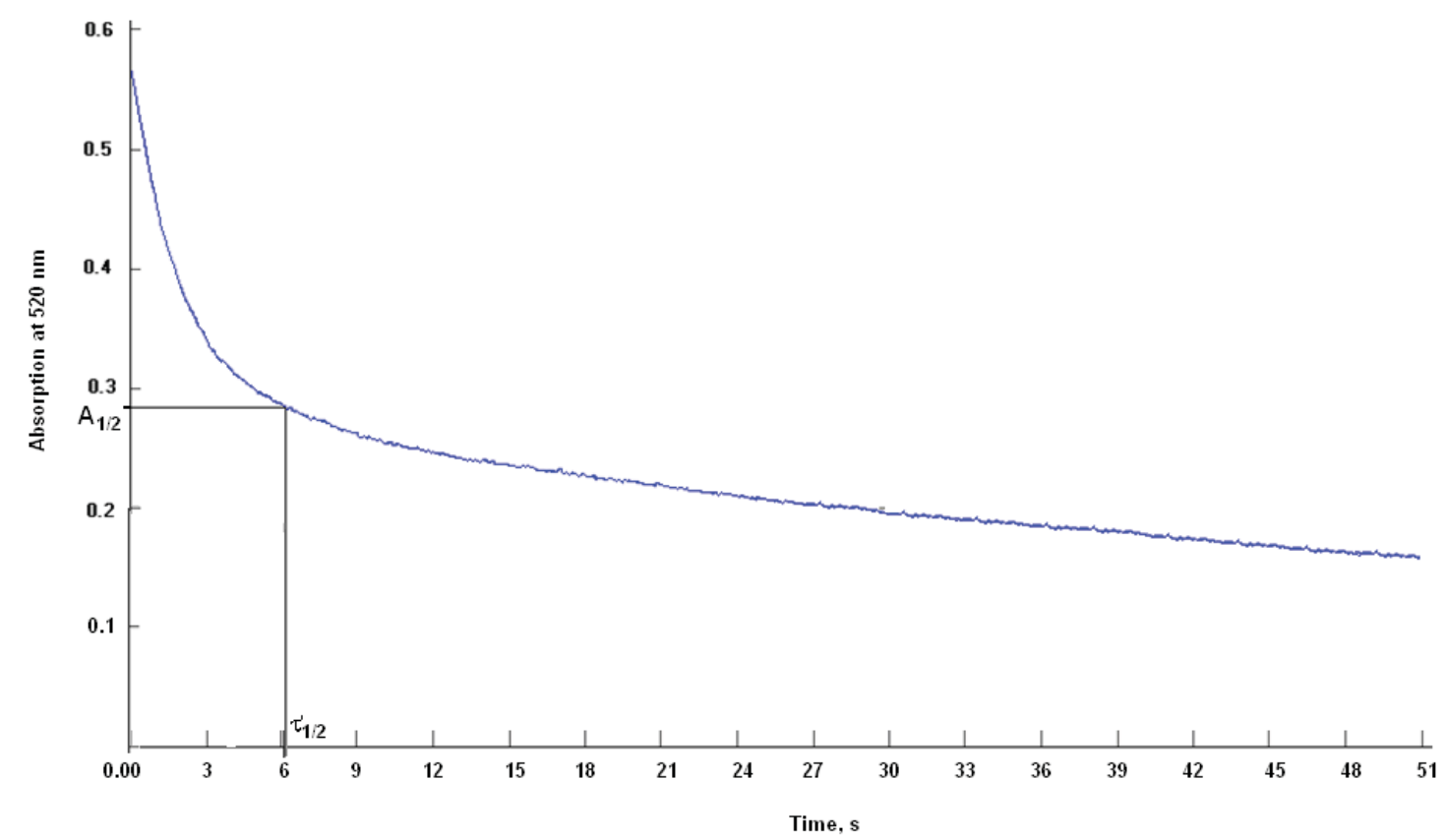

Fig.1. Time evolution of the reaction of DHF and $\mathrm{DPPH}^{*}$ when initially $\mathrm{Co}(\mathrm{DHF})=\mathrm{Co}\left(\mathrm{DPPH}^{*}\right) / \sigma$, where $\mathrm{Co}(\mathrm{DHF})=$ $3 \cdot 10^{-5} \mathrm{M}$, and $\sigma=2$.

Fig.1 shows the time evolution of the concentration of $\mathrm{DPPH}^{*}$ when it is being reduced by dihydroxyfumaric acid (in the case when initially $\left.\mathrm{Co}(\mathrm{DHF})=\mathrm{Co}\left(\mathrm{DPPH}^{*}\right) / \sigma\right)$. As it is observed, the curve which describes the $\mathrm{DPPH}^{*}$ decay represents the sum of two consecutive stages. The first stage is similar to that of ascorbic acid [8], corresponding to fast hydrogen atom transfer, while the second stage is somewhat slower. Thus, the stoichiometry of the overall reaction is the sum of two terms: $\sigma=\sigma_{\text {fast }}+\sigma_{\text {slow }}$, where $\sigma_{\text {fast }}$ and $\sigma_{\text {slow }}$ are the stoichiometric constants for the fast and slow stages of the reaction, respectively.

The rate (v) of a complete reaction between $\sigma$ moles of DPPH* and one mole of DHF as a function of time ( $t$ ) is defined by the sum of two terms, describing the fast and slow steps:

$$
v=\frac{-d C_{D P P H}}{\sigma \times d t}=k_{\text {fast }} \times C^{x}{ }_{D P P H} \times C^{y}{ }_{D H F}+k_{\text {slow }} \times C^{a}{ }_{D P P H} \times C^{b}{ }_{D H F}
$$

where $C$ are the initial concentrations, $k$ the rate constants, $x, y$ and $a, b$ the orders with respect to each reactive form. The determination of partial reaction orders is possible using the method of initial rates. Thus, in order to determine the reaction order with respect to $\mathrm{DPPH}^{*}$, the initial reaction rate is determined for various initial concentrations of DPPH', maintaining the concentration of DHF constant (yet always at least twice lower than the studied concentration of DPPH'). Using eq.1 (for the fast step):

$$
v=k_{\text {fast }} \times C^{x}{ }_{D P P H} \times C^{y}{ }_{D H F}
$$

after a logarithmic conversion and plotting $\lg (\mathrm{v})$ as a function of $\lg \left(\mathrm{C}_{\mathrm{DPPH}}\right), x$ can be determined from the slope of the linear curve.

Analogically, the reaction order with respect to DHF is determined for various initial concentrations of DHF, maintaining the concentration of DPPH' constant and at least twice greater than the studied DHF concentration. Again, plotting $\lg (\mathrm{v})$ as a function of $\lg \left(\mathrm{C}_{\mathrm{DHF}}\right), y$ can be determined from the slope of the linear curve.

Another way to determine the reaction order with respect to DHF is using eq.1 when initially $\mathrm{C}_{\mathrm{DHF}}=\mathrm{C}_{\mathrm{DPPH}} / \sigma$. Using eq.1 (for the fast step):

$$
v=\frac{-d C_{D P P H}}{\sigma_{\text {fast }} \times d t}=k_{\text {fast }} \times C_{D P P H}^{x} \times C_{D H F}^{y}
$$


and after a logarithmic conversion:

$$
\lg (\mathrm{v})=\lg \left(\mathrm{k}_{\text {fast }} / \sigma_{\text {fast }}^{\mathrm{y}}\right)+(\mathrm{x}+\mathrm{y}) \mathrm{x} \lg \mathrm{C}_{\mathrm{DPPH}}
$$

Thus, $y$ can be determined from the slope of the linear curve, since $x$ is known. Eq. 2 makes it possible to determine the rate constant $\mathrm{k}_{\text {fast }}$, since $\lg \left(\mathrm{k}_{\text {fast }} / \sigma_{\text {fast }} \mathrm{y}\right)$ is the intersection of the linear curve described by eq.2 and the ordonate axis.

\section{Results and discussion}

Experiments were carried out at $20^{\circ} \mathrm{C}$. Taking into consideration the strict requirements for reactions studied by the stopped flow method, the common DPPH* assay was slightly modified: the solvent for the DPPH' and DHF consisted of $70 \%$ methanol and $30 \%$ of bidistilled water. The solutions of DPPH' and DHF were brought to $\mathrm{pH} 4.0 \mathrm{by}$ adding acetic acid (respecting the ratio of $70 \%$ for methanol). In these experimental conditions, prior to antioxidant activity investigations, calibration curves of absorbance vs. concentration were obtained for the two substances.

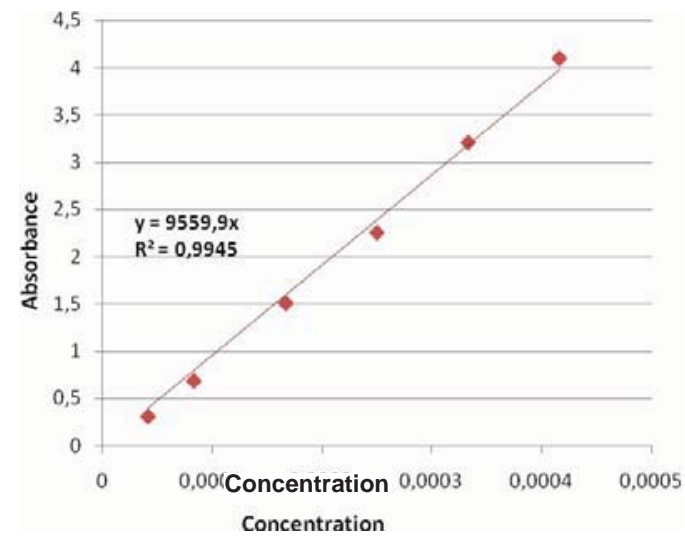

Fig.2.a) Determination of the molar extinction coefficient for the DPPH* radical in the experimental solvent

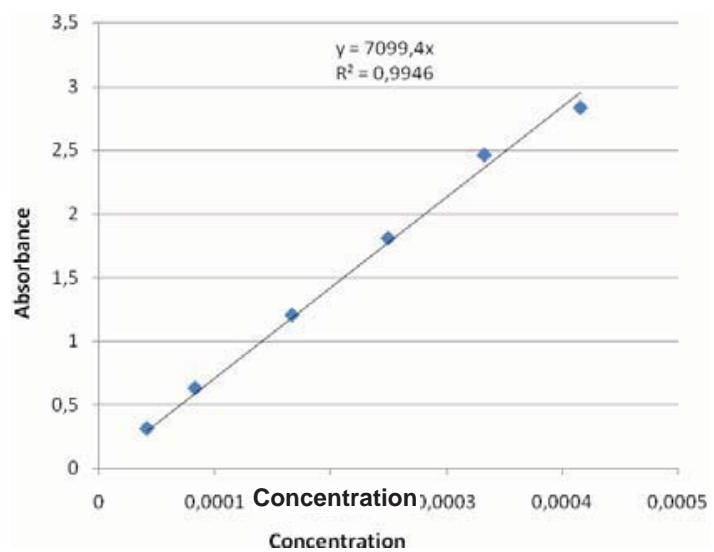

Fig.2.b) Determination of the molar extinction coefficient for the DHF in the experimental solvent

It should be mentioned that DPPH* decay was monitored at $520 \mathrm{~nm}$ (not at $515 \mathrm{~nm}$ [2] or $517 \mathrm{~nm}$ ), as in described experimental conditions, the $\mathrm{DPPH}^{*}$ absorption spectrum presented a peak at $520 \mathrm{~nm}$.

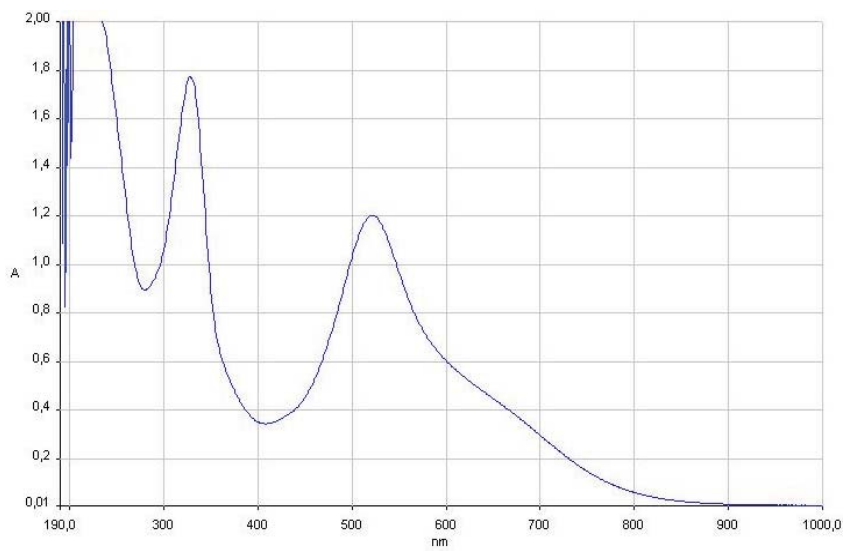

Fig.3. DPPH spectrum in $70 \% \mathrm{MeOH}$ at $\mathrm{pH} 4.0$

The exact initial concentration of $\mathrm{DPPH}^{*}$, noted $\mathrm{Co}\left(\mathrm{DPPH}^{*}\right)(\mathrm{mol} / \mathrm{L})$ was determined using the relationship $\mathrm{Co}=\mathrm{A} / \varepsilon$, where $\varepsilon$ was determined 9560 (Fig.2a). The exact initial concentration of DHF, noted Co(DHF) (mol/L) was determined using the relationship $\mathrm{Co}=\mathrm{A} / \varepsilon$, where $\varepsilon$ in $70 \%$ methanol was determined 7100 (Fig.2.b).

The obtained value for $\mathrm{EC}_{50}$ was found, according to results presented in Fig.4, $\mathrm{EC}_{50}=0.24$. Therefore, the stoichiometric constant determined for the overall reaction was $\sigma=1 /\left(2 \times \mathrm{EC}_{50}\right)=2.08$, corresponding to the number of freely available $\mathrm{OH}$ groups. 


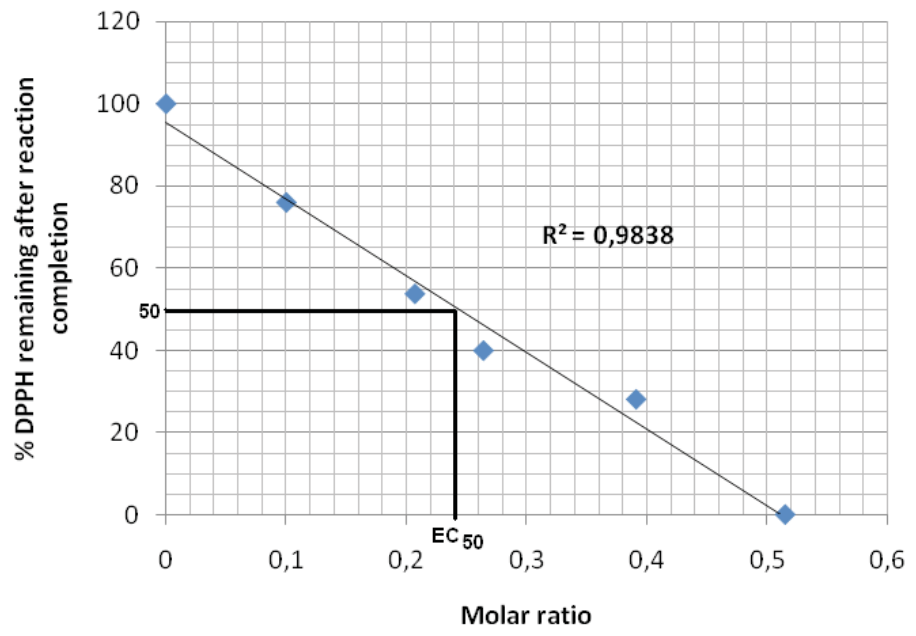

Fig. 4. Determination of the global reaction molecularity

The determined stoichiometric constants for the fast and slow stages of the reaction are $\sigma_{\text {fast }} \sim 1$ and $\sigma_{\text {slow }} \sim 1$, i.e. one molecule of DHF interacts very fast with one molecule of DPPH', undergoes transformation and afterwards interacts with a second molecule of DPPH', a lot slower. The chemical structure of dihydroxyfumaric acid suggests the following mechanism of reduction of $\mathrm{DPPH}^{*}$ :

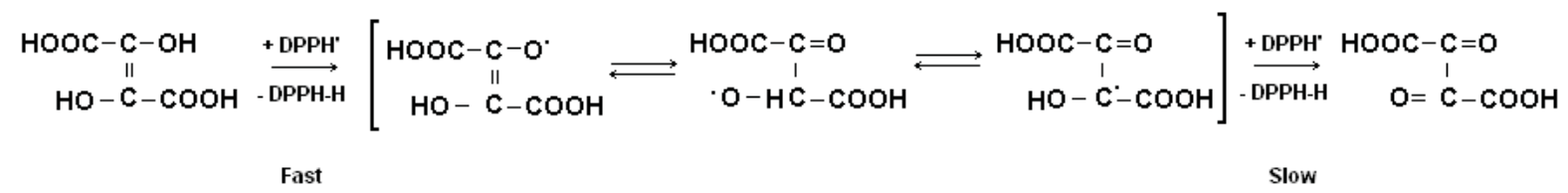

In experiments when initially $\mathrm{C}_{\mathrm{DHF}}=\mathrm{C}_{\mathrm{DPPH}} / \sigma$, the fast stage of the reaction was terminated during several seconds (Fig. 1). The reaction orders describing the fast stage of the reaction were found to be $x=1$ (Fig.5.a) and $y \sim 0.5$ (Fig.5.b), thus the rate constant was calculated $\mathrm{k}_{\text {fast }}=39,81 \mathrm{~L} \mathrm{~mol}^{-1} \mathrm{~s}^{-1}$. In classical chemical kinetics, the order 0.5 towards DHF implicates the dissociation of this compound prior to the rate determining step [9].

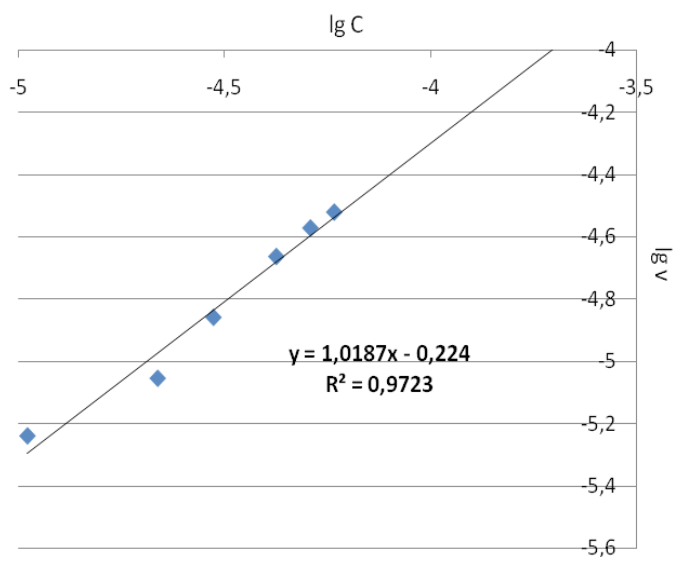

Fig.5.a) Reaction order with regards to initial DPPH* concentration

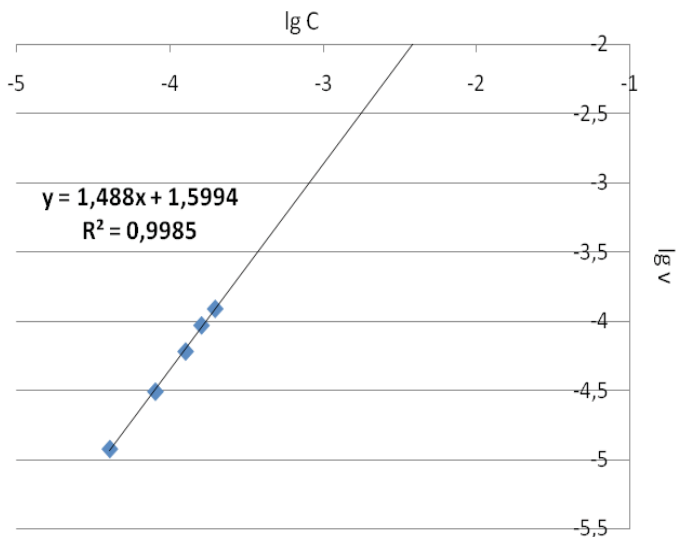

Fig.5.b) Global reaction order for the fast stage of the reaction

The reaction orders describing the slow stage of the reaction were found to be $x=0.77$ (Fig.6.a) and $y=0.28$ (Fig.6.b), thus the rate constant is $\mathrm{k}_{\text {slow }}=0.0012 \mathrm{~s}^{-1}$. 


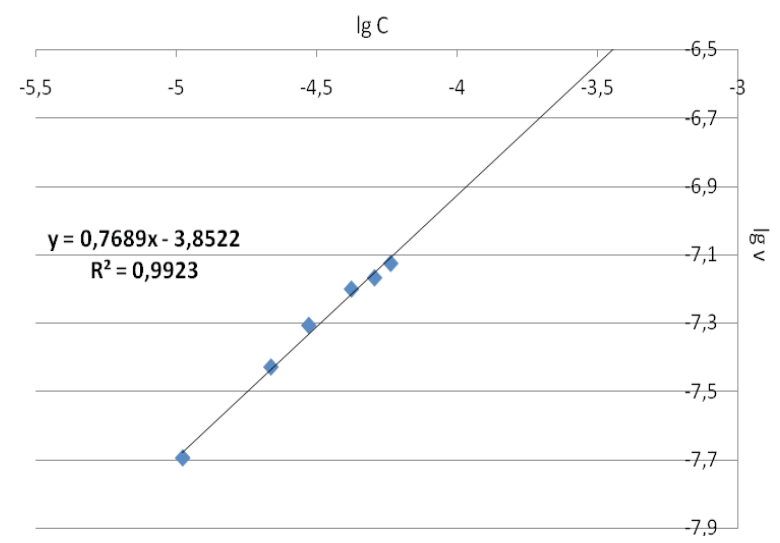

Fig.6.a) Reaction order with regards to initial DPPH* concentration

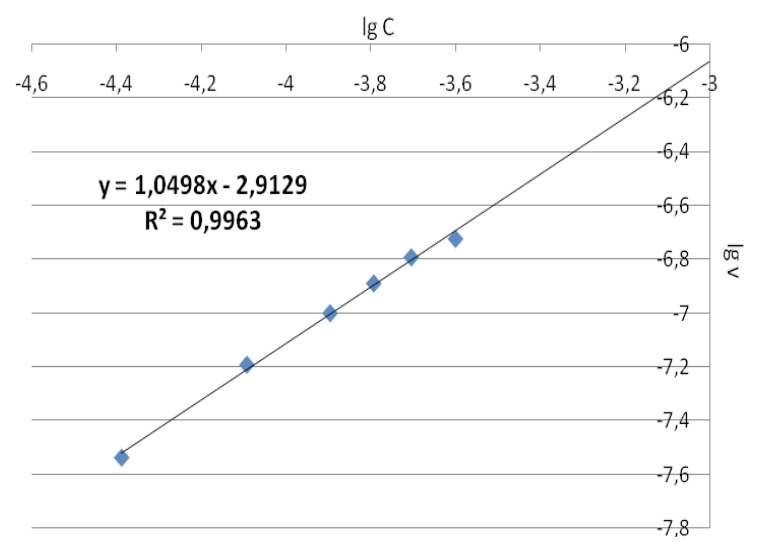

Fig.6.b) Global reaction order for the slow stage of the reaction

\section{Conclusions}

The DPPH* assay provides a rapid estimation of the antioxidant activity of chemical compounds. However, in some cases, when the reaction proceeds at a high rate, it is quite difficult (if not impossible) to estimate the reaction kinetic parameters using conventional spectrophotometry. In these cases, the use of stopped flow spectrophotometry allows researchers to get an important insight on the reaction evolution in various conditions.

The dihydroxyfumaric acid proved to have a quite good antioxidant capacity $\left(\mathrm{EC}_{50}=0.24\right)$. The utilization of stopped flow apparatus allowed us to determine that the reaction of DHF with the DPPH radical proceeds in two consecutive stages, the first being similar to the interaction of ascorbic acid with DPPH', corresponding to fast hydrogen atom transfer, while the second stage - slower. The overall determined molecularity of the reaction is 2 , and the molecularities of the fast and slow stage are each equal to 1 . The reaction orders describing the fast stage of the reaction were found to be $\mathrm{x}=1$ (Fig.5.a) and $\mathrm{y} \sim 0.5$ (Fig.5.b), thus the rate constant was calculated 39,81 L mol ${ }^{-1} \mathrm{~s}^{-1}$. The reaction orders describing the slow stage of the reaction were found to be $x=0.77$ (Fig.6.a) and $y=0.28$ (Fig.6.b), thus the rate constant of the slow stage is $0.0012 \mathrm{~s}^{-1}$.

Acknowledgements: I would like to express my sincere gratitude to my scientific adviser who helped me every step of my research.

\section{References}

[1]. Beckman Kenneth B. and Ames Bruce N., The journal of biological chemistry Vol. 272, No. 32, Issue of August 8, pp. 19633-19636, 1997

[2]. Bondet V., Brand-Williams W. and Berset C., Lebensm.-Wiss. u. -Technol., vol 30, pp.609-615, 1990

[3]. Nagy P. and Ashby M. T., Chem. Res. Toxicol. 2007, 20, 79-87

[4]. M. Valko, H. Morris and M.T.D. Cronin, Current Medicinal Chemistry, 2005, 12, 1161-1208

[5]. Ilhami G., Riad E. Akcahan G, Laurent B and Ekrem K., African Journal of Biotechnology Vol.6 (4), pp 410-418, 19 February 2007

[6]. Duca Gh., Gonta M., Mereuta A., Processing and Valorization of Secondary Winery Products, NATO Science for Peace and Security Series C: Environmental Security, Springer Netherlands, 2009, pp 197-207.

[7]. Molyneux, P. Songklanakarin J. Sci. Technol., 2004, 26(2), pp. 211.219.

[8]. Sendra, J.M., Sentandreu E., Navarro, J.L., Eur. Food Res. Technol. 2006, 223, pp. 615-624

[9]. Fremaux, B. Elements de Cinetique et de Catalyse. Paris: Lavoisier, 1989. 